\title{
Common bile duct dilatation according to the type of anastomsosis after gastrectomy for gatric cancer
}

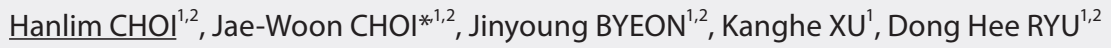 \\ 'Department of Surgery, Chungbuk National University College of Medicine, Cheongju, Korea \\ ${ }^{2}$ Department of Surgery, Chungbuk National University Hospital, Cheongju, Korea
}

Introduction: There are many studies for the increased incidence for choledocholithiasis and common bile duct (CBD) dilatation after gastrectomy. However, there are few reports about the dilatation of CBD according to the type of anastomosis in patients undergoing gastrectomy for gastric cancer. We compared the changes of CBD diameter after gastrectomy with various anastomosis. The aim of this study was to evaluate the degree of CBD dilation in patients who underwent subtotal gastrectomy with various anastomosis using 1-year follow-up abdominal computed tomography (CT) scan.

Methods: From January 2012 to December 2015, 395 patients who underwent subtotal total gastrectomy for gastric cancer and had no gallstones were enrolled. We measured CBD diameter in the pancreas head by analyzing preoperative, 6-, and 12-month follow-up abdomen-pelvis enhanced CT (A-P CT) scans.

Results: The CBD was dilated slightly from $4.1 \mathrm{~mm}$ at baseline to $5.1 \mathrm{~mm}$ at 6 months and $6.1 \mathrm{~mm}$ at 12 months after gastrectomy. The number of cases of CBD dilatation of more than $7 \mathrm{~mm}$ at 6 months and at 12 months after cholecystectomy were 11 (24.4\%) and 9 (29.0\%), respectively. Seven cases at 6 months and 5 cases at 12 months showed bile duct dilation of more than $3 \mathrm{~mm}$ compared to baseline. There were no cases having bile duct dilation of more than $10 \mathrm{~mm}$.

Conclusions: The diameter of CBD was significantly increased after subtotal or total gastrectomy. In addition, the degree of dilation of CBD was higher after not only Billroth-II with Braun anastomosis but Roux-en Y anastomosis compared. 